\title{
A Bibliometric and Visualized Analysis of Nursing Informatics Competencies in China (2000-2020)
}

\author{
Yuchao LE ${ }^{\mathrm{a}}$, Shihua $\mathrm{CAO}^{\mathrm{b}, 1}$, Mengxi WANG ${ }^{\mathrm{a}}$, Xin $\mathrm{LIN}^{\mathrm{c}}$ and Beiying QIAN \\ ${ }^{a}$ School of Medicine, Hangzhou Normal University, Hangzhou, China \\ ${ }^{\mathrm{b}}$ Qianjiang College, Hangzhou Normal University, Hangzhou, China \\ ${ }^{\mathrm{c}}$ Bengbu $3^{\text {rd }}$ Hospital, Bengbu, China
}

\begin{abstract}
This study aimed to use CiteSpace software to conduct a bibliometric analysis of published on the nursing informatics competencies from 2000 to 2020 . Data were obtained from the China National Knowledge Infrastructure and Wanfang Database. Co-occurrence analysis, collaboration analysis and Co-Words Analysis of keywords were conducted by using CiteSpace5.7 R2 software. A total of 496 publications were obtained. The number of publications generally increased over the investigated period. The most publications were from colleges and universities. 'Influencing factor', 'nursing informatics competencies', and 'nursing education' were the hotspots recently. The study may be helpful for researchers in identifying the research status quo, trend and development of nursing informatics competencies in China in the future.
\end{abstract}

Keywords. Nursing informatics competencies, CiteSpace, visual analysis, Research hotspots introduction

\section{Introduction}

With the rapid development of information technology in healthcare settings, a growing emphasis on the importance of nursing informatics has emerged. Nursing informatics is described by the American Nurses Association (ANA) "the science of nursing with information, technological, communication, and analytical sciences to support the integration of data, information, knowledge, and wisdom into the provision of evidencebased nursing care" [1]. Given the ubiquitous emphasis of nursing informatics within healthcare settings, there has been a focus on ensuring that nurses and nursing students have the informatics competencies that enable them to function effectively in nursing.

In recent years, there has been an increasing trend of research using CiteSpace in China. However, CiteSpace is not widely used in the field of nursing, even less in nursing informatics. Therefore, this study aimed to use CiteSpace5.7 R2 software to conduct a bibliometric analysis of Chinese published studies related to nursing informatics competencies from 2000 to 2020 . The study also aimed to determine growth and development of trend in this field, and to provide reference for further studied.

${ }^{1}$ Corresponding Author, Shihua Cao, Professor, Qianjiang College, Hangzhou Normal University, Hangzhou, China; E-mail: csh@hznu.edu.cn. 


\section{Methods}

Data were collected from the China National Knowledge Infrastructure (CNKI) and Wanfang Database. The data retrieval strategy included the topic (nursing AND informatics competencies) OR the topic (nursing students AND informatics competencies) OR the topic (nurse AND informatics competencies). The search period was set from 2000 to 2020, and the document type is journal. Through manual screening and data removal function of NoteExpress software, a total of 496 cases of valid literature data were finally included after screening out the conference reports, news and duplicates.

CiteSpace is the method chosen to perform the bibliometric analysis, including the analysis of the authors, institutions and trends in the identified publications. [2] The methods mainly include co-occurrence analysis, cluster analysis and burst term analysis. [3] The visualization knowledge network created by CiteSpace consists of nodes and lines. The nodes in the network stand for items and lines between the nodes represent cooperation or co-occurrence or co-citation relationships. The size of each node indicates the count. $[4,5]$

\section{Results}

\subsection{Publications Output}

A total of 496 publications were included in the study. The distribution of annual publications varied at different times (See table 1). In general, research interest on nursing informatics competencies has increased significantly in recent years, as the number of publications from 2 in 2000, to 54 in 2020 .

Table 1. The number of annual publications on nursing informatics competencies between 2000-2020.

\begin{tabular}{cc||cc}
\hline Years & $\begin{array}{c}\text { Number of annual } \\
\text { publications }\end{array}$ & Years & $\begin{array}{c}\text { Number of annual } \\
\text { publications }\end{array}$ \\
\hline 2000 & 2 & 2011 & 29 \\
2001 & 1 & 2012 & 32 \\
2002 & 4 & 2013 & 24 \\
2003 & 4 & 2014 & 38 \\
2004 & 11 & 2015 & 38 \\
2005 & 9 & 2016 & 41 \\
2006 & 7 & 2017 & 36 \\
2007 & 17 & 2018 & 38 \\
2008 & 12 & 2019 & 50 \\
2009 & 23 & 2020 & 54 \\
2010 & 26 & & \\
\hline
\end{tabular}

\subsection{Distribution of Institution}

There were 352 institutions which contributed to a total of 496 publications. The top ten most prolific institutions which published on nursing informatics competencies were shown in Table 2 and figure 1. The institutions with most publications were the Jilin University Nursing College, Changsha Medical University Nursing College, Zhengzhou University Nursing College and Xiangtan Medicine \& Health Vocational College, respectively. 
Table 2. The top ten institutions in terms of publications about the nursing informatics competencies between 2000-2020.

\begin{tabular}{ccl}
\hline Rank & Publications & \multicolumn{1}{c}{ Institution } \\
\hline 1 & 4 & Jilin University Nursing College \\
1 & 4 & Changsha Medical University Nursing College \\
1 & 4 & Zhengzhou University Nursing College \\
1 & 4 & Xiangtan Medicine \& Health Vocational College \\
2 & 3 & Hubei University of Arts and science \\
2 & 3 & Xiangya School of Nursing School of Central South University \\
2 & 3 & Hangzhou Normal University Nursing College \\
2 & 3 & University of south China school of nursing \\
2 & 3 & School of Public Health Jilin University \\
2 & 3 & The First Affiliated Hospital of Guangxi Medical University
\end{tabular}

University of south China school of nursing

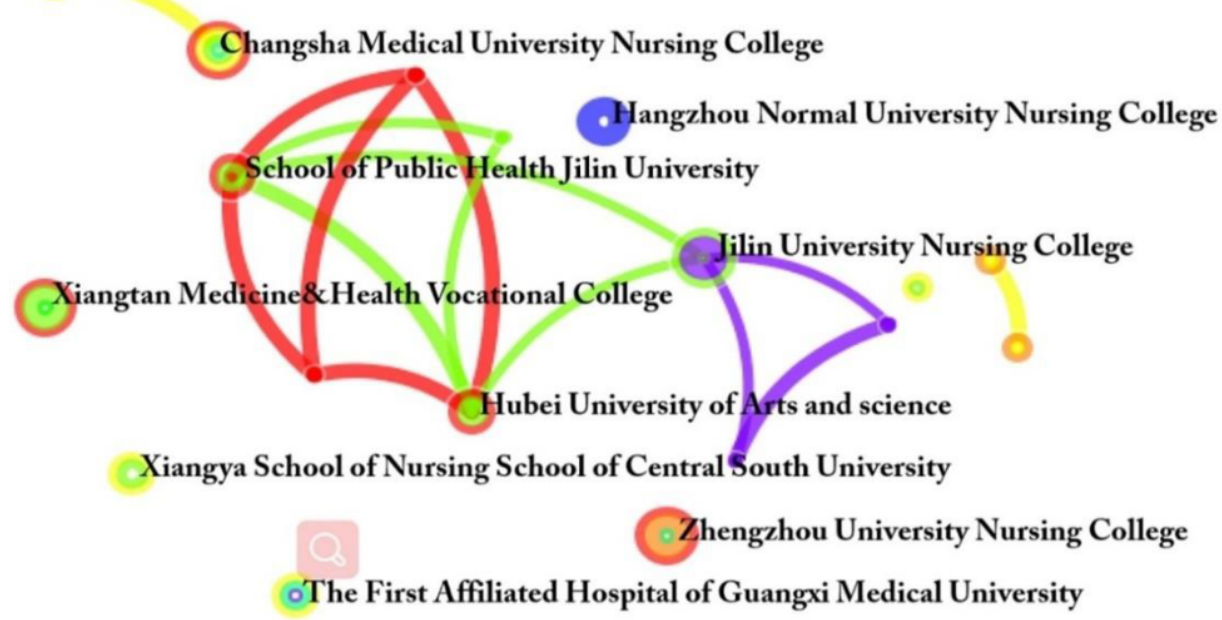

Figure 1. Top ten institutions in terms of co-occurrence counts. The nodes represent institutions, and lines between the nodes represent collaborative relationships.

\subsection{Co-Words Analysis of Keywords}

The keywords associated with an increased burst in citation, which frequently appeared within a certain period, were considered to be indicators of frontier topics or emerging trends. [6] Figure 2 showed the top 10 keywords with the strongest citation bursts between 2000-2020. 


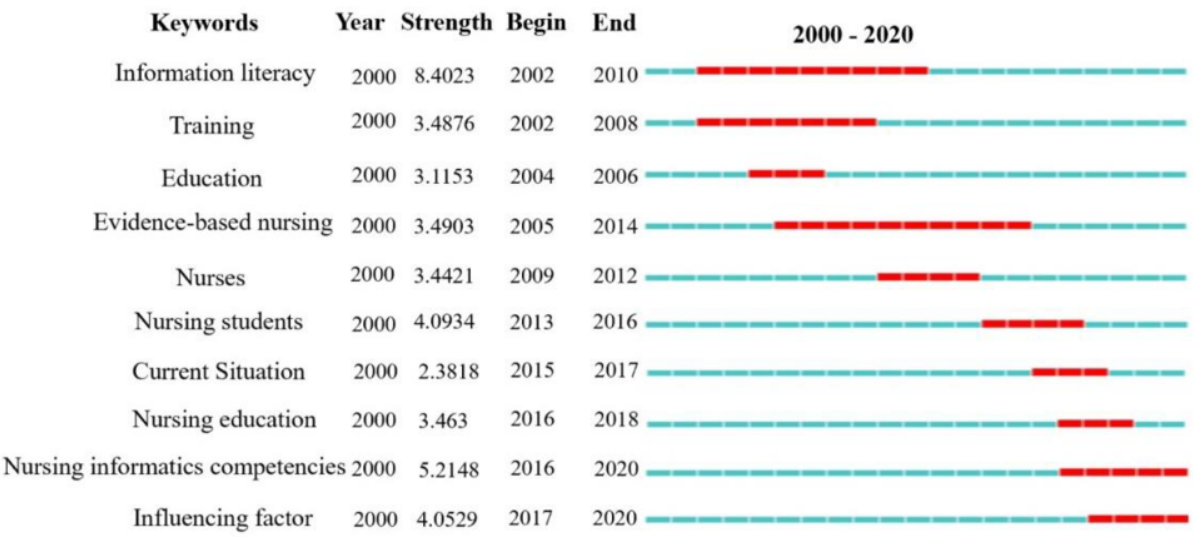

Figure 2. The keywords with the strongest citation bursts for publications on nursing informatics competencies between 2000-2020. The red bars represent frequently cited keywords. The green bars represent infrequently cited keywords.

\section{Discussion}

With rapid growth of healthcare information systems and technology, [7] much importance is attached to the training of nursing informatics competencies for nursing students and nurses. [8-10] According to the annual publications, the overall trend in publications in this field showed a pattern from 2000-2008, where annual publications increased slowly, and from 2009-2020, where annual publications in this field increased rapidly.

Analysis by institution identified that there was only one hospital in the top 10 institutions in terms of Publications about the nursing informatics competencies, and nine of the top ten most prolific institutions were from the colleges and universities. This finding was also reflected that the colleges and universities were research leaders in this filed in China. Meanwhile, there were a few lines between the nodes in the figure 1. It showed five of the top ten academic institutions conducted cooperation with other institutions. Therefore, more cooperation between core institutions and teams may be needed. Burst keywords were considered to be indicators of frontier topics or emerging trends. In this study, the earlier burst keywords included 'information literacy' and 'training'. The keyword 'Nursing students' and 'Nursing informatics competencies' also had a strong burst, respectively, suggesting that nursing informatics competencies of nursing students have been concerned by more and more researchers. A few studies to date have reported nursing students' informatics competencies and patient safety competencies were significantly correlated [11,12], maybe that's why nursing informatics competency has become increasingly important in education of nursing students.

\section{Conclusion}

This Study aimed to use CiteSpace5.7 R2 software to conduct a bibliometric analysis of published studies retrieved from CNKI and Wanfang database about nursing informatics 
competencies from 2000 to 2020 . The results could help investigators to realize core institutions, determine the status of research topics and identify new direction for future in the field. The findings showed that the number of publications on nursing informatics competencies has been increasing, the most publications were from colleges and universities. Analysis of keywords identified 'influencing factor', 'nursing informatics competencies', and 'nursing education' as terms that may represent emerging areas of research in this filed.

\section{Limitations}

In this study, data was only retrieved from CNKI and Wanfang database. Therefore, the analysis may limit the generalizability of the results. Besides, the search criteria defined the topic of the study, but we cannot guarantee that each publication was completely relevant to the topic. However, we believe that this study can still be used to describe the overall situation and general trend in this field.

\section{References}

[1] American Nursing Association. Nursing informatics [EB/OL]. https:/www.nursingworld.org/nursesbooks/nursing-informatics-scope-and-standards-of-practice-2nd-ed/. Accessed October 25, 2018

[2] Synnestvedt MB, Chen C, Holmes JH. CiteSpace II: Visualization and knowledge discovery in bibliographic databases [J]. AMIA Annu Symp Proc, 2005: 724-8.

[3] Jingqi D, Xin Z, Zhichao Z et al. Visual analysis of research hotspots in nursing informatics in the past ten years [J]. Chinese Journal of Modern Nursing, 2020, 26(17): 2256-2260.

[4] Yue C, Chaomei C, Zhigang H. Principles and applications of analyzing a citation space [M]. Bei Jing: Science Press, 2014.

[5] Eck N, Waltman L. Visualizing bibliometric networks [J]. Springer International Publishing, 2014.

[6] Xu C, Yanyi L. Knowledge graph of nursing information based on CiteSpaceV bibliometric analysis in China [J]. Journal of Modern Nursing, 25(1): 19-25.

[7] Lulu X, Jian D, Ying Y. The research tendencies of medical informatics and the transformation toward health informatics since 2000 [J]. Journal of the China Society for Scientific and Technical Information, 2020, 39(7): 777-786.

[8] Zijuan Y, Yan Z. Reliability and validity of Chinese version of self-assessment of nursing informatics competencies scale [J]. Chongqing Medicine, 2019, 48(2): 267-269.

[9] Hong L, Xiaohan L, Yuling L et al. Development and test of reliability and validation of Nursing Informatics Competency Scale [J]. Information Management, 2020, 20(3): 423-427.

[10] Rong X, Meijuan C. The importance and training strategies of nursing information competencies among undergraduate nursing students [J]. Chinese Journal of Nursing Education, 2012, 9(2): 67-69.

[11] Abdrbo AA. Nursing informatics competencies among nursing students and their relationship to patient safety competencies: Knowledge, attitude, and skills. Comput Inform Nurs. 2015 Nov;33(11):509-14.

[12] Johansson PE, Petersson GI, Nilsson GC. Nursing students' experience of using a personal digital assistant (PDA) in clinical practice - an intervention study. Nurse Educ Today. 2013 Oct;33(10):124651. 